\title{
PMDV-hop: An effective range-free 3D localization scheme based on the particle swarm optimization in wireless sensor network
}

\author{
Wenjuan Wang ${ }^{1}$, Yuwang Yang ${ }^{1}$, Lei Wang ${ }^{2}$ and Wei Lu ${ }^{1}$ \\ ${ }^{1}$ School of Computer Science and Engineering, Nanjing University of Science and Technology \\ ${ }^{2}$ School of Computer Science and Technology, Nanjing university of posts and telecommunications \\ Nanjing -CHINA \\ [e-mail: yuwangyang@mail.njust.edu.cn] \\ *Corresponding author: Yuwang Yang
}

Received June 17, 2016; revised November 2, 2017; accepted November 6, 2017;

published January 31, 2018

\begin{abstract}
Abstract: Location information of individual nodes is important in the implementation of necessary network functions. While extensive studies focus on localization techniques in 2D space, few approaches have been proposed for 3D positioning, which brings the location closer to the reality with more complex calculation consumptions for high accuracy. In this paper, an effective range-free localization scheme is proposed for 3D space localization, and the sensitivity of parameters is evaluated. Firstly, we present an improved algorithm (MDV-Hop), that the average distance per hop of the anchor nodes is calculated by root-mean-square error (RMSE), and is dynamically corrected in groups with the weighted RMSE based on group hops. For more improvement in accuracy, we expand particle swarm optimization (PSO) of intelligent optimization algorithms to MDV-Hop localization algorithm, called PMDV-hop, in which the parameters (inertia weight and trust coefficient) in PSO are calculated dynamically. Secondly, the effect of various localization parameters affecting the PMDV-hop performance is also present. The simulation results show that PMDV-hop performs better in positioning accuracy with limited energy.
\end{abstract}

Keywords: Range-free localization, sensitivity of parameters, improved algorithm, intelligent optimization algorithms. 


\section{Introduction}

In wireless sensor networks (WSNs), localization has received a great deal of attention since it facilitates a variety of applications such as environmental monitoring, health care, target tracking, and military surveillance [1][2]. This technique is also considered to be a fundamental requirement especially when the nodes are randomly distributed to the rugged environment, in which the network topology is uneven and unpredictable [3]. Thus, in this case, it becomes more difficult for nodes to obtain the location information owing to the intricate environment. While, the location information is crucial important for nodes realizing the surrounding environment characteristics. It shows that more effective localization algorithms need to be exploited for 3D applications [4-6].

In many practical applications, 3D space is very complex, rendering the irregularity. Such as when the nodes spread to the forest plants hierarchical structure, the distribution of nodes becomes more complicated. However, the range-free localization algorithm essentially depends on the location information of anchor nodes to locate other nodes, the anchor nodes own vital effects on the accuracy of algorithm [7]. In recent researches, the common key external factors in node location include the following points. (i) Independent unknown nodes, having no connectivity and no communication with other nodes, they can't be located [8]. (ii) Independent anchor nodes, no connectivity and no communication with other nodes, locate itself with GPS, while not belongs to the 3D WSNs for locating other unknown nodes [9]. (iii) Independent nodes group, group of nodes without any communication to other group of nodes, so they make no contributions to node positioning. Except for these factors, the anchor ratio also has certain influence on the performance of localization algorithms [10]. In other words, the higher anchor ratio is, the better position the localization algorithm performs. As a result, the effective distribution of anchor nodes can not only improve the position precision, but also can reduce the ratio of anchor nodes, and reduce the localization cost.

In addition to the external factors, there is some other internal factors in node localization which are inevitable in the implementation of localization algorithm. For varied algorithms, the internal factors would be different. According to the range-free localization algorithms [11][12], the factors affected node positioning accuracy can be divided into the following procedures:

- The average value of the estimated distance per hop: the irregular network topology of 3D space, the path for nodes transferring messages becomes more twists and turns. Besides, unknown nodes obtain the estimated value from anchor nodes and calculate the distance with the hops [13[14], as a result that the average value of the estimated distance per hop becomes inaccuracy with even larger deviations.

- The calculation method of node location: there are many varied calculation methods for node location, among which the trilateration measurement method and the iterative method of multilateral are widely used in node positioning [15-17]. The former one has low computing complexity and implement which is only based on three anchor nodes as reference. However, this method would lead to high inaccuracy positioning. In contrast, the latter one can improve the positioning accuracy with complex calculation. 
- The anchor nodes in special position: there exist two conditions that anchor nodes and unknown nodes are in special locations [18], so that they are useless for node positioning. One condition is that all anchor nodes are in one plane. The other condition is that all anchor nodes are in one line. So the special location of anchor nodes indeed has much effect on the positioning accuracy.

The remainder of the paper is organized as follows: section 2 discusses related works. Section 3 introduces network models and DV-Hop algorithm in node positioning. Section 4 presents an improved 3D localization algorithm based on DV-Hop (MDV-Hop), and proposes an effective range-free localization scheme based on improved PSO (PMDV-Hop). Section 5 presents simulation results to evaluate the performance of all the proposed localization algorithms. Finally, section 6 concludes and analyzes the work in this paper.

\section{Related Work}

There are many works related to wireless networks. Yang Liu et al.[34-35] have many works in varied wireless networks, for example, Mobile Ad Hoc Social Networks and Mobile Opportunistic Networks. In contrast to their network, this paper focus on wireless sensor networks(WSNs), which consist of many sensor nodes that communicate wireless to monitor a physical region. In wireless sensor networks, location information is critical essential and indispensable for many applications of WSN. Recently, various localization schemes have been proposed, while most research proposals are designed and evaluated only considering 2D applications, where the sensing area is assumed flat and node deployment is assumed dense enough. However, the real sensing area may have a complex terrain and large altitude differences. Such as, sensor nodes may be deployed in a mountainous battlefield for surveillance or be suspended in the air for pollution monitoring. In such applications, it is unreliable only to simplify the localization problem to 2D level. As a result, the 3D localization problem in WSNs poses new challenges for localization scheme design.

A 3D accurate positioning algorithm based on RSSI [19], which carries out parameter matching for wireless signal attenuation model between every pair of beacon node and unknown node, and then makes a compensation for the loss of wireless signal using theoretical and empirical models when there are obstacles (walls) between them. Cheng et al. [20] proposed a novel underwater localization algorithm for sparse 3D acoustic sensor networks, which transformed 3D underwater localization to 2D. Chen et al. [21] enhanced conventional centroid localization algorithm in 2D space to 3D, which uses a large number of anchor nodes with their localization accuracy inversely proportional to the number of anchor nodes employed. Besides, the rang-free algorithm DV-HOP is also extended to 3D space by Wang L et al. [22]. Shi et el. [23] introduced a 3D localization approach for WSNs. their localization approach employs a mobile beacon, which emits Ultra-wideband (UWB) signals to achieve location positioning. Upon reception of the UWB signals, each sensor node measures the distance to the mobile anchor using the TOA technique.

Particle swarm optimization (PSO) is a popular multidimensional optimization technique [24], which was introduced by Kennedy and Eberhart in 1995. Ease of implementation, high quality of solutions, computational efficiency, and speed of convergence are strengths of the PSO. As for the applications of PSO in WSNs localization, PSO-Loc for localization of $n$-target nodes out of $m$ nodes based on the a priori information of locations of $m-n$ beacons has been proposed by Gopakumar et al. [25], and Kulkarni et al. [26] have proposed a distributed iterative localization algorithm PSO-Iterative. For each target node, there exists 
three or more beacons in its hearing range and PSO is exploited to minimize the localization error. Low et al. [27] have proposed a PSO-based distributed-localization scheme that does not involve beacons in which nodes are deployed by an unmanned aerial vehicle equipped with a position sensor. Low et al. [28] have proposed PSO-4 Beacon localization scheme in which the target node uses PSO to find the best value of $\alpha$ Kalman-filter-based recursive estimation to localize itself. Meanwhile, research on performance improvements, including parameter studies, combination with auxiliary operations, and topological structures, has been reported in [29].

Motivated by above observations, our research attempts to get an effective localization scheme for 3D WSNs localization. Among the distributed localization algorithms, DV-hop is a neat scheme without considerring the error from distance measurement method. Hence, for further investigation in 3D node positioning, DV-hop is the preferred algorithm to be exploited among all the range-free localization techniques. For more localization accuracy, PSO algorithm is also introduced. In a word, this paper proposes a novel effective scheme with improved PSO algorithm based on DV-hop technique. Our contributions can be summarized as follows:

We propose a localization algorithm (MDV-Hop) based on dynamic error correction and multi-hop scheme according to the DV-Hop localization algorithm. Further to improve the accuracy of node positioning in 3D space, an improved particle swarm optimization of intelligent optimization algorithm is expanded to MDV-Hop localization algorithm which is called PMDV-Hop. The effect of various parameters affecting the performance of proposed algorithm is also presented. The efficiency of the proposed algorithm is established through the comparison simulations of four localization algorithms including DV-hop, MDV-hop, PDV-hop and PMDV-hop.

\section{Network model and DV-hop algorithm in 3D space}

\subsection{Network model}

The localization definition: give a multihop network $\mathrm{G}=(\mathrm{V}, \mathrm{E})$, and a set of beacon nodes and their positions $\left(x_{i}, y_{i}, z_{i}\right)$, we want to find the position of unknown nodes $(\mathrm{x}, \mathrm{y}, \mathrm{z})$ as many as possible , then employ these nodes to locate other unknown nodes.

In localization network $\mathrm{G}=(\mathrm{V}, \mathrm{E})$ there are varied models for localization. Fig. 1 shows typical network models of 3D space localization. In these models, at least four anchor nodes are required to locate unknown nodes, and all the four anchor nodes can't be in the same line or the same plane. Fig. 1(a) shows the case that one unknown node $D$ can be located by the four anchor nodes ( $A, B, C, D$ ). The second case shown in Fig. 1(b) indicates that four anchor nodes ( $A, B, C, D)$ in the same plane could calculate two potential positions $(E, F)$. In Fig. 1(c), four anchor nodes are in the same line, the exact position cannot be located because all the positions $\left(\alpha_{1}, \alpha_{2}, \cdots, \alpha_{\mathrm{n}}\right)$ in the circle may be the right position. 


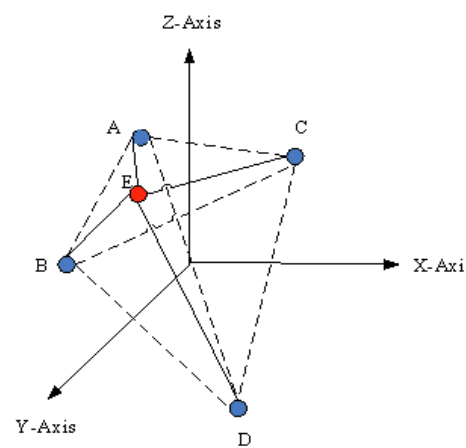

(a) Typical network mode

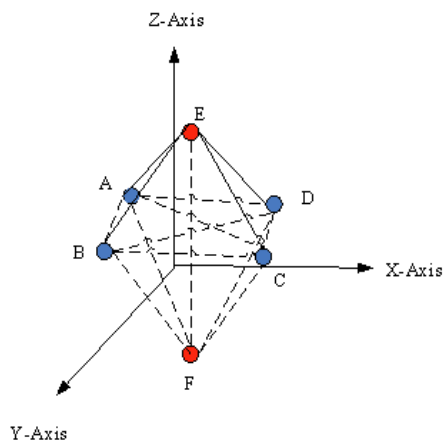

(b) Four anchor nodes in one plane

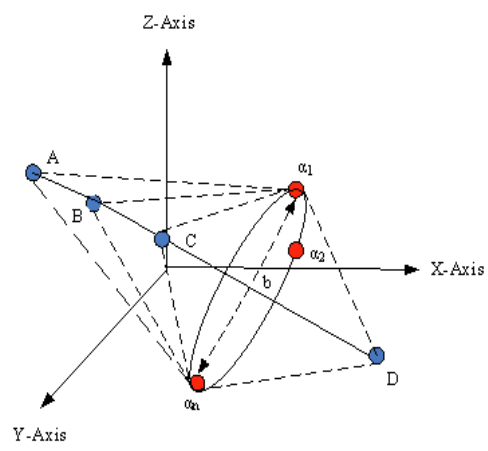

(c) Four anchor nodes in one line

Fig. 1. The Localization Network Models in 3D Space

\subsection{DV-Hop algorithm in 3D space}

This section describes conventional DV-Hop algorithm. In the algorithm simulation, a large number of sensor nodes are deployed in 3D space, in which there exist two types of nodes, normal nodes and anchor nodes [30]. The normal nodes own identical sensing, processing and communication capabilities with the same battery power, and the anchor nodes are equipped with GPS receiver except for the characteristics of normal nodes. Meanwhile, all the nodes are static, and , the DV-Hop algorithm is mainly divided into the following steps:

First step, anchor nodes flood and broadcast their position information to adjacent nodes. The receiving nodes select the message owning the smallest hop information among all the messages sent by neighbor anchor nodes, and transfer the message to neighbors with the hop added one count. As a result, the minimum hop to anchor nodes is updated. From Fig. 2, it can be observed that the smallest hop of unknown node Node to the anchor nodes Anchor ${ }_{1}$, Anchor ${ }_{2}$, Anchor 3 , Anchor ${ }_{4}$, Anchor $r_{5}$ are 5, 3, 4, 4, 1 respectively.

Second step, according to Eq. (1), the average distance per hop is calculated with the minimum hop to the other anchor nodes and their obtained localization information. Meanwhile, the message containing the average distance per hop would be broadcasted to network, some messages selectively discarded, owing to that unknown nodes only receive the message from the nearest anchor node, it can be assured that the anchor node is the closest node.

$$
\operatorname{Hop}_{i}=\frac{\sum_{i \neq j} \sqrt{\left(x_{i}-x_{j}\right)^{2}+\left(y_{i}-y_{j}\right)^{2}+\left(z_{i}-z_{j}\right)^{2}}}{\sum_{i \neq j} h_{i j}}
$$

Here, $\left(x_{i}, y_{i}, z_{i}\right)$ are the coordinates of anchor node $i$. And the other anchor nodes can be signed as $\left(x_{j}, y_{j}, z_{j}\right) . h_{i j}$ is the minimum hop of anchor node $i$ to another node $j$. In Fig. 2, assumed that the coordinates of anchor nodes are obtained and the distance between anchor nodes Anchor ${ }_{1}$ Anchor $_{5}$, Anchor Anchor $_{5}$, Anchor Anchor $_{5}$, Anchor Anchor $_{5}$ are $56 \mathrm{~m}, 52 \mathrm{~m}, 32 \mathrm{~m}$, 47m respectively. And the minimum hop of Anchor $_{5}$ to the other anchor nodes are 6, 4, 3, 4 separately, the average distance per hop of Anchor $_{5}$ is $(56+52+32+47) /(6+4+3+4)=11 \mathrm{~m}$. 


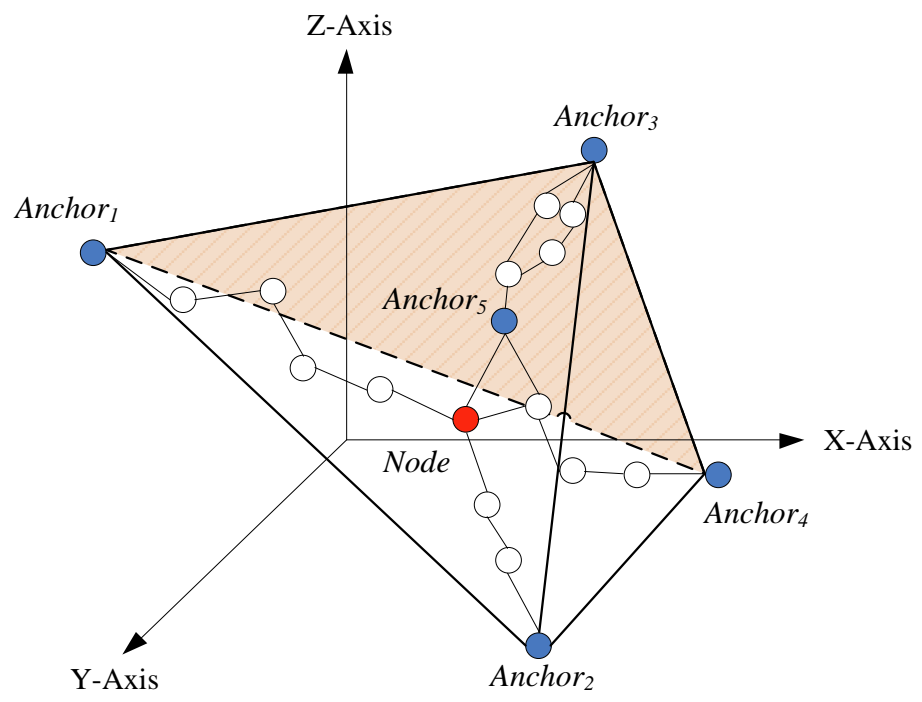

Fig. 2. Schematic Diagram of DV-Hop Localization Algorithm

Third step, according to the obtained hop distance and minimum hop, the distance of

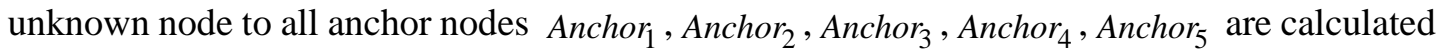
as $d_{a 1}=5 \times 11=55 \mathrm{~m}, d_{a 2}=3 \times 11=33 \mathrm{~m}, d_{a 3}=4 \times 11=44 \mathrm{~m}, d_{a 4}=4 \times 11=44 \mathrm{~m}, d_{a 5}=1 \times 11=11 \mathrm{~m}$, respectively.

Fourth step, assumed that the coordinate of unknown node is $(x, y, z)$, the coordinates of anchor nodes are $\left(x_{1}, y_{1}, z_{1}\right),\left(x_{2}, y_{2}, z_{2}\right), \ldots,\left(x_{n}, y_{n}, z_{n}\right)$, and the estimated distance of unknown node to anchor nodes are $d_{1}, d_{2}, \ldots, d_{n}$, Eq. (2) can be obtained and simplified to Eqs. (3)-(5) according to the formula $A X=b$. As a result, $A, b$ and $X$ can be acquired based on Eqs. (3)-(5) respectively. So the coordinate of unknown node can be derived as: $X=\left(A^{T} A\right)^{-1} A^{T} b$.

$$
\begin{aligned}
& \left\{\begin{array}{c}
\sqrt{\left(x-x_{1}\right)^{2}+\left(y-y_{1}\right)^{2}+\left(z-z_{1}\right)^{2}}=d_{1} \\
\sqrt{\left(x-x_{2}\right)^{2}+\left(y-y_{2}\right)^{2}+\left(z-z_{2}\right)^{2}}=d_{2} \\
\vdots \\
\sqrt{\left(x-x_{n}\right)^{2}+\left(y-y_{n}\right)^{2}+\left(z-z_{n}\right)^{2}}=d_{n}
\end{array}\right. \\
& A=\left\{\begin{array}{ccc}
2\left(x_{1}-x_{n}\right) & 2\left(y_{1}-y_{n}\right) & 2\left(z_{1}-z_{n}\right) \\
2\left(x_{2}-x_{n}\right) & 2\left(y_{2}-y_{n}\right) & 2\left(z_{2}-z_{n}\right) \\
\ldots & \\
2\left(x_{n-1}-x_{n}\right) & 2\left(y_{n-1}-y_{n}\right) & 2\left(z_{n-1}-z_{n}\right)
\end{array}\right. \\
& b=\left[\begin{array}{c}
x_{1}^{2}-x_{n}{ }^{2}+y_{1}{ }^{2}-y_{n}{ }^{2}+z_{1}{ }^{2}-z_{n}{ }^{2}+d_{n}{ }^{2}-d_{1}{ }^{2} \\
x_{2}{ }^{2}-x_{n}{ }^{2}+y_{2}{ }^{2}-y_{n}{ }^{2}+z_{2}{ }^{2}-z_{n}{ }^{2}+d_{n}{ }^{2}-d_{2}{ }^{2} \\
\vdots \\
x_{n-1}{ }^{2}-x_{n}{ }^{2}+y_{n-1}{ }^{2}-y_{n}{ }^{2}+z_{n-1}{ }^{2}-z_{n}{ }^{2}+d_{n-1}{ }^{2}-d_{n}{ }^{2}
\end{array}\right]
\end{aligned}
$$




$$
X=\left[\begin{array}{l}
x \\
y \\
z
\end{array}\right]
$$

\section{Effective range-free localization scheme}

\subsection{MDV-Hop: improved localization scheme based on DV-Hop}

In accordance with the above section, the estimated distance per hop has vital effect in localization application, we proposed a scheme in order to improve the localization accuracy. As Fig.3, node $s$ receives average distance per hop of anchor nodes $a_{1}, a_{2}, b_{1}, b_{2}, b_{3}, b_{4}, b_{5}, c_{1}, c_{2}, c_{3}, c_{4}, c_{5}, c_{6}$, as well anchor nodes with the same distance to node $s$ are in one group. With the distance per hop in each group calculated, the estimated distance to the unknown node can be obtained. From Fig. 3, it is observed that node $a_{1}, a_{2}$ are in same group marked $G_{1}$ in one hop to node $s$. Similarly, $b_{1}, b_{2}, b_{3}, b_{4}, b_{5}$ and $c_{1}, c_{2}, c_{3}, c_{4}, c_{5}, c_{6}$ are marked into group $G_{2}$ and group $G_{3}$ respectively in two hops and four hops to node $s$.

For the grouped anchor nodes, firstly, the average distance per hop is adjusted dynamically with its distance to unknown node and the average distance error per hop. Secondly, the weighted value can be calculated based on the RSME of anchor nodes, meanwhile, the distance per hop in one group is the sum of all the distance per hop of anchor nodes with weighted value. Finally, the distance of the unknown node to anchor node can be computed with the distance per hop of unknown nodes in varied groups. For node $s$, the distance to anchor nodes in this group can be estimated with the distance per hop in $G_{1}$ group. Similarly, the step is executed in $G_{2}$ and $G_{3}$.

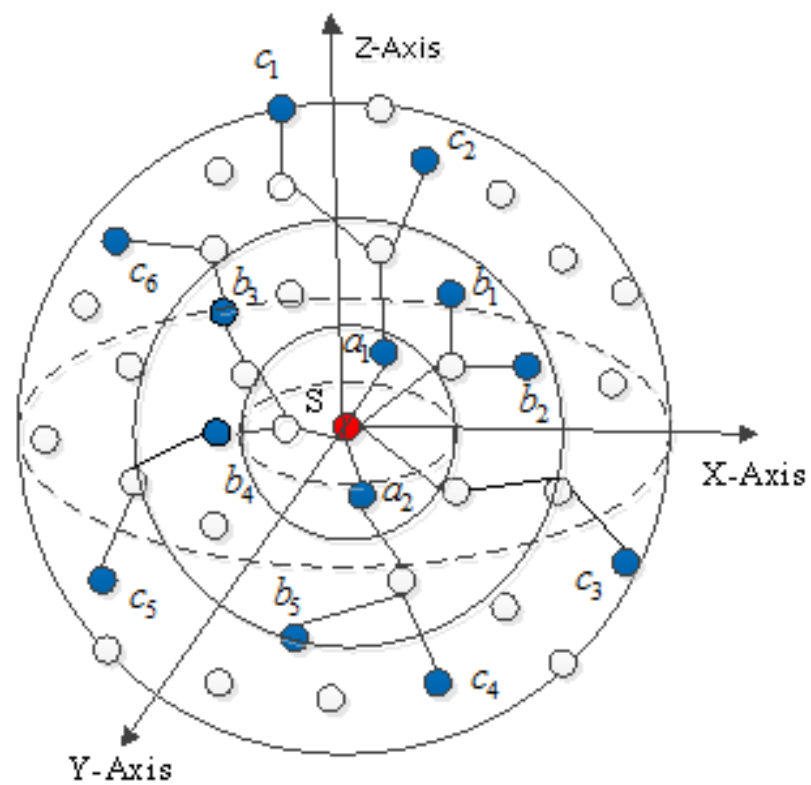

Fig. 3. Multipath Schematic Diagram based on DV-Hop Localization Algorithm 
Step 1: in DV-Hop localization algorithm, assumed that there exist $m$ anchor nodes in which the coordinates of one anchor node and the other anchor nodes are $\left(x_{i}, y_{i}, z_{i}\right)$ and $\left(x_{j}, y_{j}, z_{j}\right)$ respectively, the distance of one anchor node to the other anchor nodes is calculated as Eq. (6) and the RMSE value is obtained based on Eq. (7).

$$
\begin{array}{r}
d i s_{i j}=\sqrt{\left(x_{i}-x_{j}\right)^{2}+\left(y_{i}-y_{j}\right)^{2}+\left(z_{i}-z_{j}\right)^{2}} \\
f_{1}(i)=\sqrt{\frac{\sum_{i \neq j}\left(d i s_{i j}-H o p_{i} \times h_{i j}\right)^{2}}{m-1}}
\end{array}
$$

Here, $m-1$ is the number of the other anchor nodes as reference. $j$ varies from 1 to $m . h_{i j}$ is the minimum hop of the anchor node to the other anchor nodes. Hop is the average distance per hop of this anchor node. For the best error evaluation, $\mathrm{Hop}_{i}$ can be calculated based on Eqs. (8)-(9), and average distance of anchor nodes on Eq. (10).'

$$
\begin{aligned}
& 2 f_{1}\left(\operatorname{Hop}_{i}\right) f_{1}^{\prime}\left(\operatorname{Hop}_{i}\right)=\frac{\sum_{i \neq j}\left(-2 d i s_{i j} h_{i j}+2 h_{i j}^{2} H_{o p}\right)}{m-1} \\
& f_{1}^{\prime}\left(H o p_{i}\right)=\frac{\sum_{i \neq j}\left(h_{i j}^{2} H o p_{i}-d i s_{i j} h_{i j}\right)}{\sqrt{m-1} \times \sqrt{\sum_{i \neq j}\left(d i s_{i j}-H o p_{i} \times h_{i j}\right)^{2}}} \\
& \left\{\begin{array}{c}
\operatorname{Hop}_{i}=\frac{\sum_{i \neq j} d i s_{i j} h_{i j}}{\sum_{i \neq j} h_{i j}^{2}} \\
\text { Hop }_{i} \neq \frac{d i s_{i j}}{h_{i j}}
\end{array}\right.
\end{aligned}
$$

Step 2: In multipath schematic diagram, the coordinates of anchor node and the others are denoted as $\left(x_{i}, y_{i}, z_{i}\right)$ and $\left(x_{j}, y_{j}, z_{j}\right)$, separately. Based on the average distance per hop of anchor node $i, H_{i}$ calculated with the minimum hop and the anchor node $i$ to the other anchor nodes signed as $h_{i j}$, then the estimated distance between them can be calculated according to Eq. (11). Meanwhile, the average error per hop and the RSME value are obtained based on Eq. (12) and Eq. (13) respectively.

$$
\begin{gathered}
d i s_{i j}^{\prime}=H o p_{i} \times h_{i j} \\
\varepsilon_{i}=\frac{\sum_{i \neq j}\left|d i s_{i j}^{\prime}-d i s_{i j}\right| / h_{i j}}{m-1}
\end{gathered}
$$




$$
r_{i}=\sqrt{\frac{\sum_{i \neq j}\left(d i s_{i j}-H o p_{i} \times h_{i j}\right)^{2}}{m-1}}
$$

Here, $m$ is the number of anchor nodes and the distance of anchor node $i$ to the other anchor nodes is $d i s_{i j}$.

Step 3: Anchor node $i$ broadcasts a message containing the average distance error per hop and RMSE information to unknown nodes, and update the average value per hop in each group dynamically. Assumed that there exist $k$ groups of anchor nodes related to unknown node $s$, in which the hop to the kth group is $h_{s i}$ and the hop to the furthest anchor node is $h_{k_{-} \text {max }}$, the hop of the $k$ th anchor node group can be updated as follows.

MDV-Hop algorithm: the first improved procedure on DV-Hop

Requirement: The matrix containing the distance per hop, the distance error per hop and root mean square error(RMSE) information that anchor node broadcasts received by each unknown nodes, and the hop number that unknown node to each anchor nodes has been preserved in nodes

\section{1:// Adjust the distance per hop of anchor nodes dynamically}

2:Back up the group message each unknown nodes receives that anchor nodes broadcast the second time

3:Choose the biggest hop to the anchor node

4:For each anchor node

5: $\quad$ If the hop value $=1$

6: $\quad$ Keep the distance value per hop without any adjustment

7: $\quad$ Else

8: $\quad$ Adjust the distance value per hop based on the formula $H o p_{i}-e^{\varphi} \times \varepsilon_{i} \% \varepsilon_{i}$ is the

RMSE value in the preserved matrix and $\varphi$ is $\varphi=-h_{s i} / h_{k_{-} \text {max }}$, here, $i$ ranges

from 1 to the number of anchor nodes in kth group. $h_{s i}$ is the minimum hop and $h_{k_{-} \max }$ is the maximum hop in one group

9: $\quad$ End

10: End

Step 4: obtain the weighted RSME value as following MDV-Hop algorithm. Here, the updated distance per hop of $i$ node in $k$ th group is denoted as $h o p_{k_{-} i}$. The number of anchor nodes in $k$ th group is $q$ and the weighted value of each anchor nodes is $\mu_{k_{-} i}$. 


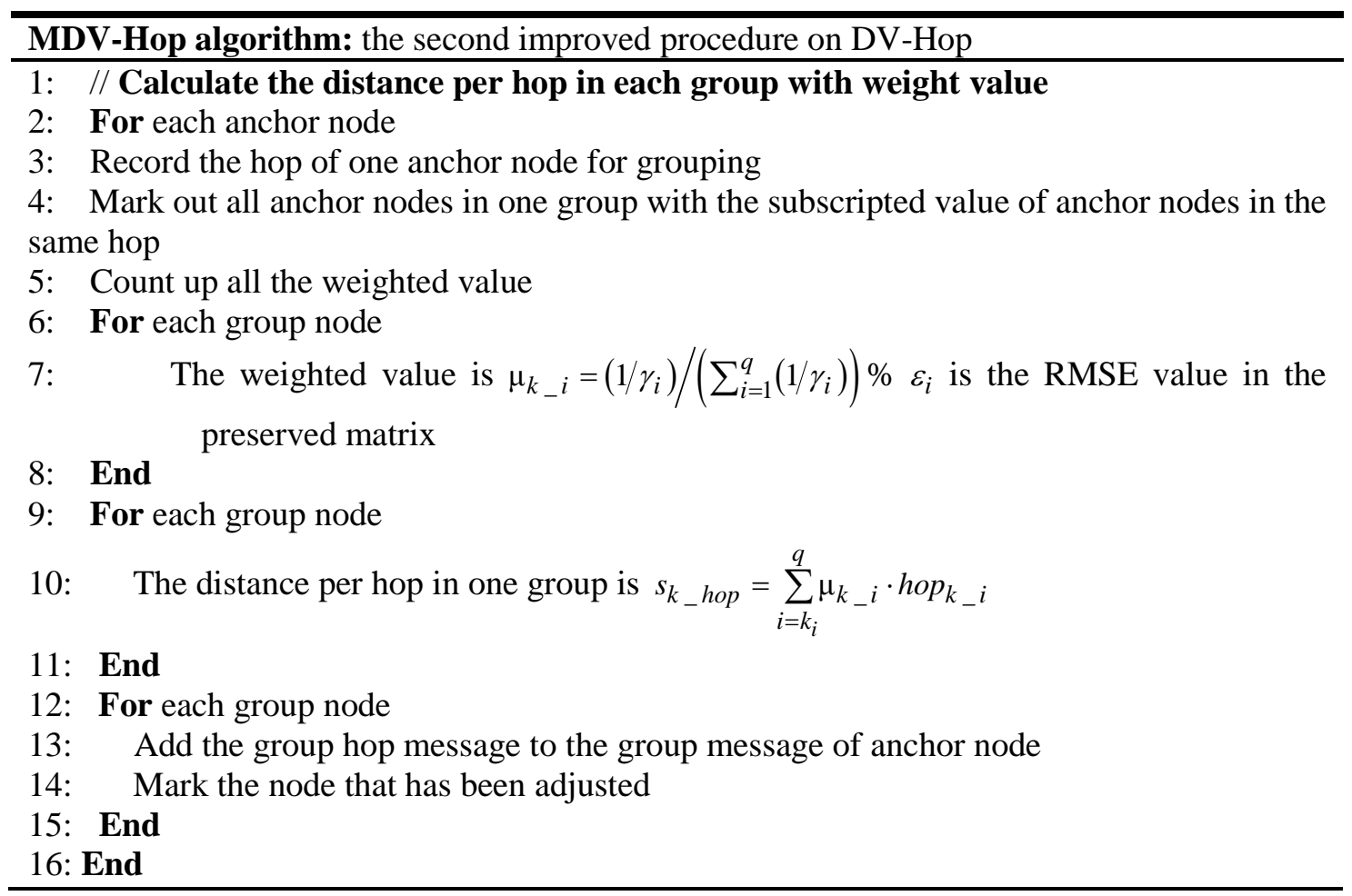

The estimated distance of unknown node $s$ to anchor nodes can be calculated as Eq.(14). Then the unknown node can be located by quadrilateral algorithm.

$$
d i s_{k_{-} i}=s_{k_{-} h o p} \times h_{k_{-} i}
$$

\subsection{PMDV-hop: An effective range-free localization scheme based on PSO}

PSO is one of the most important swarm intelligence paradigms, whose main idea is to improve social information sharing among particles in a swarm. In PSO algorithm, each individual is called a particle, and each particle moves around in D-dimensional search space with a velocity constantly updated by the particle's own experience and the experience of the best particle (which may be a neighbor). All of the particles have fitness values, which are evaluated by the objective function to be optimized, and they also have velocities. At each iteration $\mathrm{t}$, each particle keeps memory for best solution.

A. Conventional PSO algorithm

In our simulation, the number of particle swam is $s$ in the D-dimensional search space, and the particle swam is randomly distributed among $\left[x_{\min }, x_{\max }\right]$ with initial position $x_{i d}$. Here $i \in 1, \ldots, S$ and $d=1, \ldots, D$. The speed of each particle is $v_{i d}$ distributed among $\left[v_{\min }, v_{\max }\right]$. The position information and the current speed of the ith particle are $x_{i}=\left(x_{i 1}, x_{i 2}, \ldots, x_{i D}\right)$ and $v_{i}=\left(v_{i 1}, v_{i 2}, \ldots, v_{i D}\right)$ respectively. Meanwhile, the optimal history position of the ith particle and the optimal location of the whole particle swarm are $p_{i}=\left(p_{i 1}, p_{i 2}, \ldots, p_{i D}\right)$ and $p_{g}=\left(p_{g 1}, p_{g 2}, \ldots, p_{g D}\right)$ separately.

The ith particle speed of $k$ th generation in D-dimensional search space can be updated as Eq. (15). 


$$
v_{\text {id }}(k+1)=w v_{i d}(k)+c_{1} \operatorname{rand}_{i d}(?)\left[p_{\text {id }}(k)-x_{i d}(k)\right]+c_{2} \operatorname{rand}_{\text {id }}(\cdot)\left[p_{\text {gd }}(k)-x_{\text {gd }}(k)\right]
$$

Where $w$ is the inertia weight and varies in $[0,1], c_{1}$ and $c_{2}$ are the acceleration coefficients (nonnegative constant), and $\operatorname{rand}_{i d}(\cdot)$ are random numbers independently generated within $[0,1]$.

Based on Eq. (16), the location of ith particle can be updated. Once each particle moves, the optimum location would be calculated as Eq. (17). If all particles swarm finish their movement, the optimum location of whole particle swam will be computed according to Eq. (18).

$$
\begin{gathered}
x_{i d}(k+1)=x_{i d}(k)+v_{i d}(k+1) \\
p_{i}(k+1)=\left\{\begin{array}{c}
p_{i}(k), \text { ?if } f\left[x_{i}(k+1)\right] \geq f\left[p_{i}(k)\right] \\
x_{i}(k+1), \text { if } ? f\left[x_{i}(k+1)\right]<f\left[p_{i}(k)\right]
\end{array}\right. \\
p_{g}(k+1)=\arg \min _{p_{i}(k+1)} f\left[p_{i}(k+1)\right], \mathrm{P} \leq i \leq S
\end{gathered}
$$

B. Improved PSO algorithm

In PSO algorithm, the global search ability and local search ability are vital factor for optimization of algorithm performance. In order to balance the global search ability and local search ability of particles, we propose an improved Inertia weight and trust coefficient scheme of concave function.

There are many varied ways to obtain the value dynamic inertia weight. Here, the strategy of nonlinear inertia weight reduction is exploited for the inertia weight according to concave function as Eq. (19).

$$
w=\left(w_{\text {fir }}-w_{\text {last }}\right)\left(t_{\text {cur }} / t_{\text {max }}\right)^{2}+\left(w_{\text {last }}-w_{\text {fir }}\right)\left(2 \times t_{\text {cur }} / t_{\text {max }}\right)+w_{\text {fir }}
$$

Here, $w_{\text {fir }}$ is the initial inertia weight. When $t=0, w$ is the larger inertia weight $w_{\text {fir }}$. When $t=t_{\max }, w$ is the smaller value $w_{\text {last }}$.

According to Eq. (19), $c_{1} \operatorname{rand}_{i d}(\cdot)$ and $c_{2}$ rand $_{i d}(\cdot)$ have a great influence on the speed of particle swarm. When $c_{1}$ and $c_{2}$ are small, the trajectory of the particle swarm is smooth and it would be allowed to find a better solution themselves until they are back to original area. When the values are a bit larger, the particle swarm owns a larger acceleration, it can search the solution in the discrete. Usually, $c_{1}$ and $c_{2}$ are assigned constant values, so that algorithm convergent or divergent earlier. According to that, a scheme is proposed that trust coefficient dynamically changes with time and is calculated based on Eq. (20) and Eq. (21).

$$
\begin{gathered}
c_{1}= \begin{cases}c_{1} \times \frac{t_{\text {max }}-t_{\text {cur }}}{t_{\text {max }}}, & c_{1}>c_{\text {min }}: \\
c_{\text {min }} & , c_{1} \leq c_{\text {min }}\end{cases} \\
c_{2}= \begin{cases}c_{2} \times \frac{t_{\text {max }}+t_{\text {cur }}}{t_{\text {max }}}, & c_{2}<c_{\text {max }} \\
c_{\text {max }} & , c_{2} \geq c_{\text {max }}\end{cases}
\end{gathered}
$$


It is assumed that there exist $m$ anchor nodes in D-dimensional space, the coordinates of unknown node and anchor nodes are $(x, y, z)$ and $A\left(x_{1}, y_{1}, z_{1}\right), A\left(x_{2}, y_{2}, z_{2}\right), \ldots, A\left(x_{i}, y_{i}, z_{i}\right)$ respectively. And the estimated distance of unknown nodes to anchor nodes are $d_{1}^{\prime}, d_{2}^{\prime}, \ldots, ? d_{i}^{\prime}$, the formula based on the RSME is as Eq. (22).

$$
f_{(x, y, z)}=\sqrt{\frac{1}{m} \sum_{i=1}^{n}\left(\sqrt{\left(x-x_{i}\right)^{2}+\left(y-y_{i}\right)^{2}+\left(z-z_{i}\right)^{2}}-d_{i}^{\prime}\right)^{2}}
$$

Here, when the objective function is the minimum that means $(x, y, z)$ is the coordinate closest to the actual position of unknown node. And the pseudo code of PMDV-hop algorithm is shown as follows.

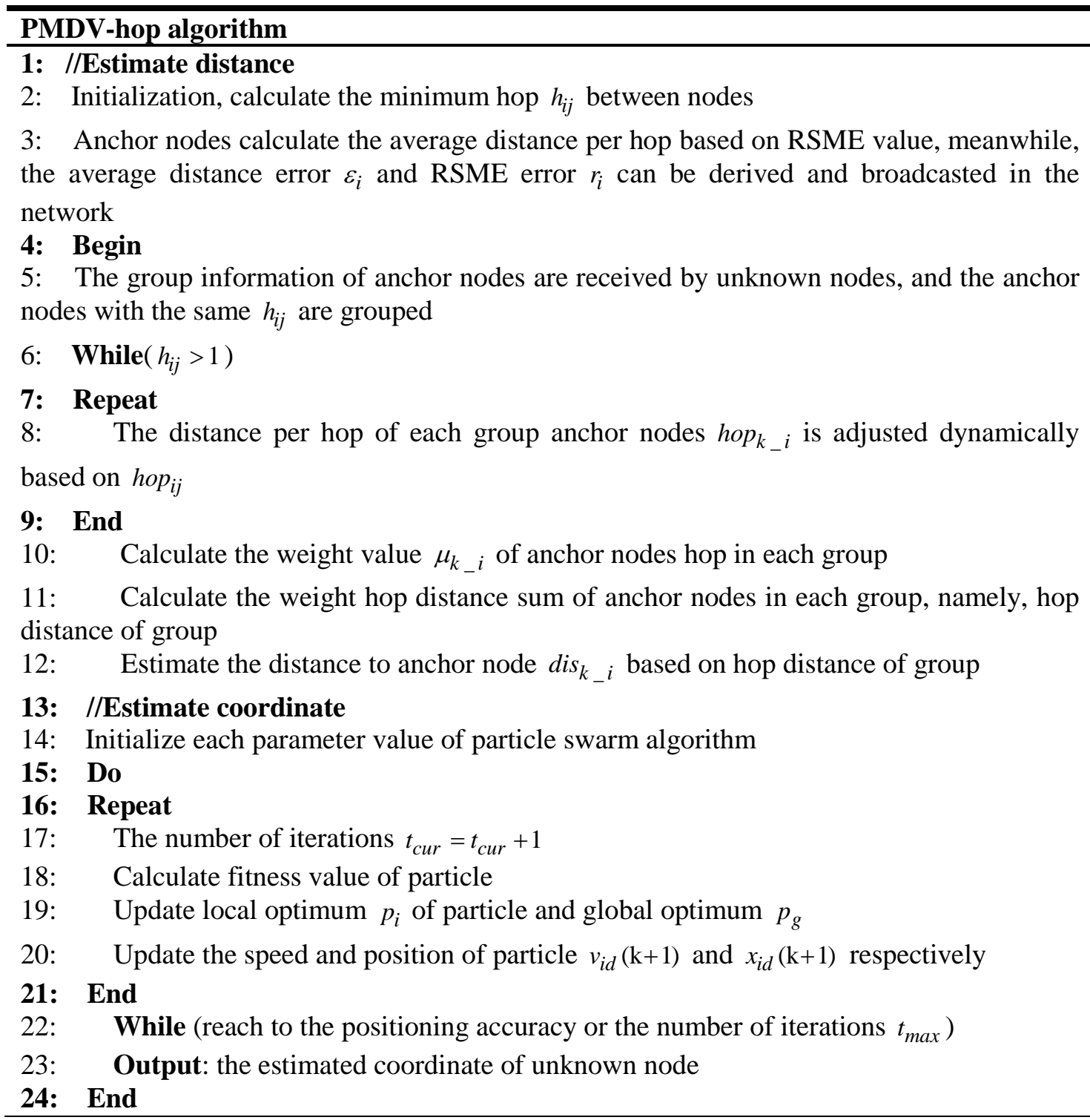




\section{Sensitivity of localization parameters}

This section provides a comprehensive analysis of our improved algorithm performance in $3 \mathrm{D}$ environment through extensive MATLAB simulations. Relevant simulation parameters are outlined below and listed in Table 1.

- The network consists of nodes varied from 80 to 280 (including anchor node ratio varied from $5 \%$ to $30 \%$ ) randomly deployed in a $3 \mathrm{D}$ region with a size of 100*100*100 units.

- Communication range varies from $20 \mathrm{~m}$ to $60 \mathrm{~m}$ to control the connectivity of the network.

- The outcomes of all simulations are averaged over 300 network instances based on the controlling variables method.

Table 1. Each parameters initialization of PMDV-hop algorithm

\begin{tabular}{cccc}
\hline Parameter & Initialization value & Parameter & Initialization value \\
\hline$D$ & 3 & $c_{1}$ & 2 \\
$S$ & 20 & $c_{2}$ & 0.5 \\
$w_{\text {fir }}$ & 0.95 & $t_{\text {max }}$ & 20 \\
$w_{\text {last }}$ & 0.4 & $x_{i d}$ & Randomly \\
$c_{\text {min }}$ & 0.5 & $v_{i d}$ & Randomly \\
$c_{\text {max }}$ & 2 & $p_{i}$ & initialize $p_{i}$ based on \\
& & & \\
$p_{g}$ & the fitness of particle & \\
& the optimum value of & & \\
\hline
\end{tabular}

As shown in Fig. 4, * represents anchor nodes, ·signifies unknown nodes, $\times$ denotes the estimated coordinate of unknown nodes.

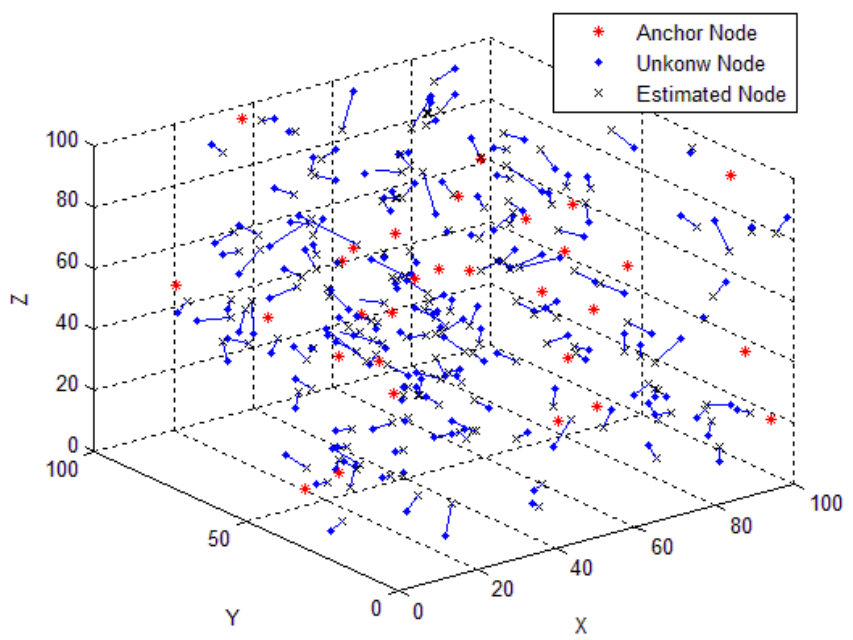

Fig. 4. True and Estimated Position after Localization for 30 Anchor Nodes and 200 Unknown Nodes 


\subsection{With varied anchor node ratio}

To begin with, the average localization error (ALE) with respect to the anchor node ratio varied from $5 \%$ to $30 \%$ is investigated in the WSNs. Herein, the number of unknown nodes is fixed to 200 and the communication range is $30 \mathrm{~m}$. As observed from Fig. 5, we can observe the average localization error is reduced as the anchor node ratio increases. Wherein, the localization error scheme is smaller than the conventional localization with a smooth gap varied from 0.64 to 0.81 . In addition, the localization error comes to be paralleled, so the improved algorithm based on the particle is steady in 3D space localization.

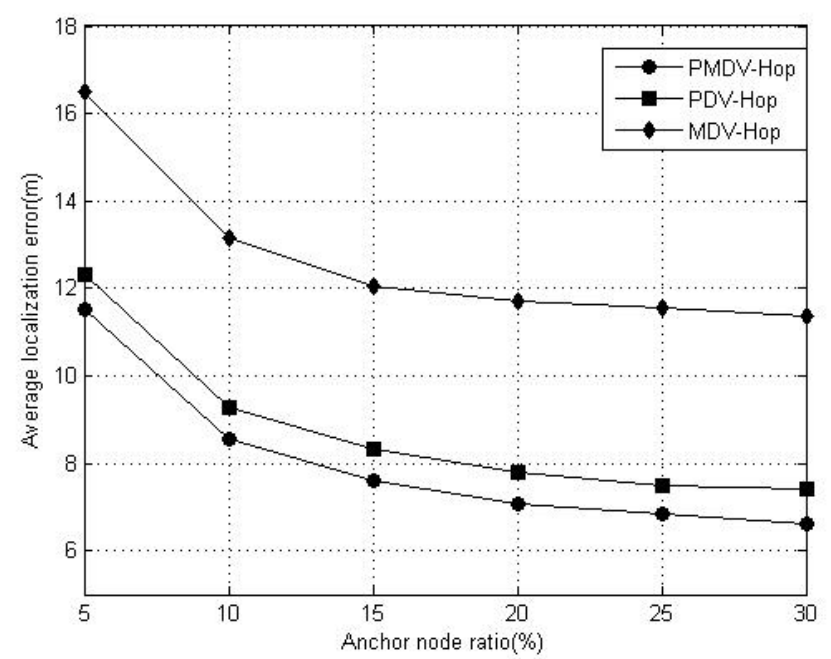

Fig. 5. Comparison of MDV-hop, PDV-hop and PMDV-hop for Varied Anchor Node Ratio in Simulation.

\subsection{With varied number of nodes}

In this section, Average localization error of normalization (ALEN) with respect to the number of unknown nodes varied from 80 to 280 is assessed in WSNs. Herein, anchor node ratio is $15 \%$ and the communication range is $30 \mathrm{~m}$. From Fig. 6, it is observed that our algorithm is fairly stable with the number of unknown nodes varied. ALEN is reduced with the number of nodes increased. If the number is less than 160, ALEN changes greater. On the contrary, as the number is more than 160, ALEN value is stable. Thus we conclude the best localization performance can be obtained when the number of nodes is 160 . Contemporary, we detect when the number of nodes is varied, ALEN is decreased as $3.64 \% 、 1.76 \% 、 2.50 \%$ 、 $2.47 \% 、 2.30 \% 、 2.57 \%$ in comparison with conventional localization algorithms. In conclusion, the improved algorithm is more stable with higher localization accuracy in 3D space. 


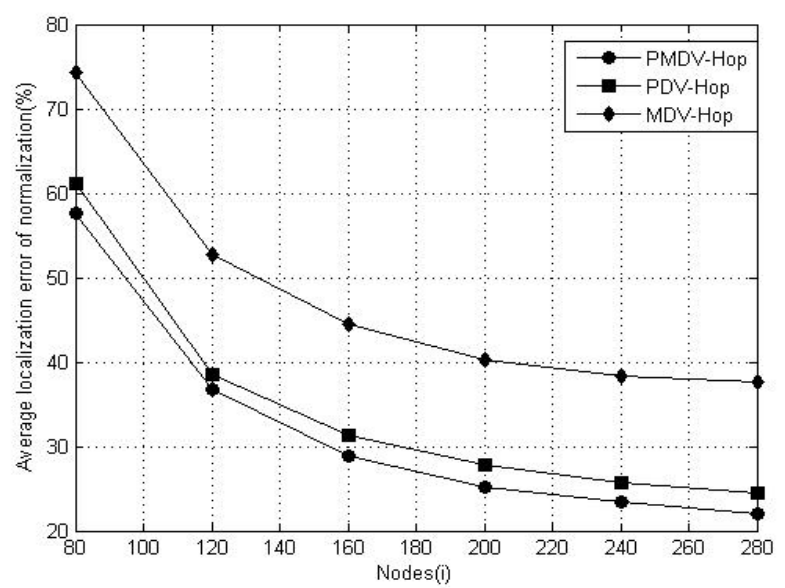

Fig. 6. Comparison of MDV-hop, PDV-hop and PMDV-hop for Varied Number of the Nodes used in Simulation.

\subsection{With varied communication range}

For the sensitivity of varied communication range, the average localization error (ALE) with respect to the communication range varied from $20 \mathrm{~m}$ to $60 \mathrm{~m}$ is simulated in the WSNs. Herein, anchor node ratio and the number of nodes is $15 \%$ and 200, respectively. From Fig. 7, we observed that our algorithm perform fairly stable for the varied communication range, while algorithms perform best at the specific range of $30 \mathrm{~m}$. When the transmission range is less than $30 \mathrm{~m}$, ALE is reduced, once more than $30 \mathrm{~m}$, the error is increased. When the communication range is $20 \mathrm{~m}$, the change of ALE is due to that more nodes would turn to isolated nodes; nevertheless, if the communication range is $40 \mathrm{~m}$, the location error becomes relative bigger for that the nodes will directly communicate with each other. The hop turns smaller so that the distance error per hop becomes bigger. From the results, we infer that the communication range of $30 \mathrm{~m}$ to $40 \mathrm{~m}$ is appropriate choice for the better evaluation of localization algorithms. Meanwhile, the both algorithms are paralleled with a fixed gap. In other words, the improved algorithm is steady in the positioning evaluations.

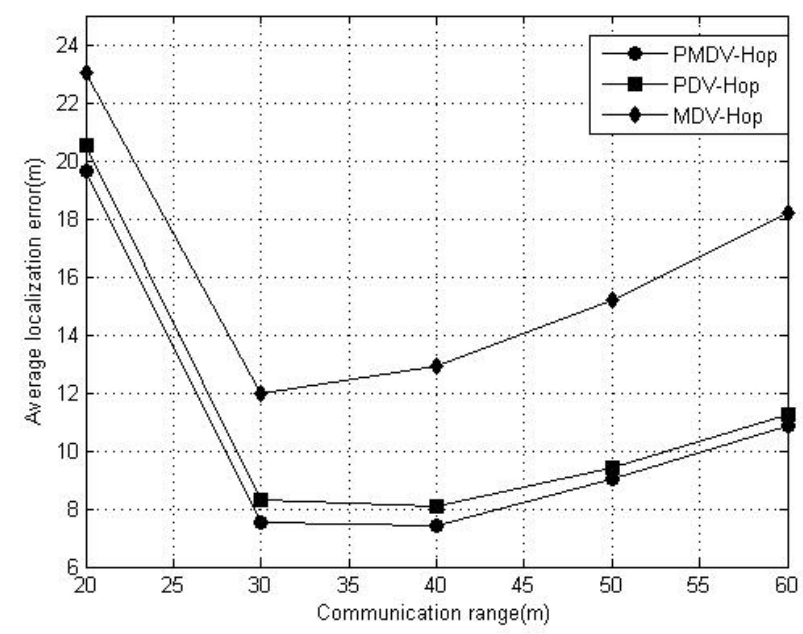

Fig. 7. Comparison of MDV-hop, PDV-hop and PMDV-hop for Varied Communication Range of the Nodes used in Simulation. 


\subsection{The comparison of 3D localization algorithms on accuracy}

The evaluation results are shown in Fig. 8, which intuitively shows the comparison of each coordinate error simulated by four localization algorithms with the same experimental parameters, i.e. the number of nodes is 120 , the anchor node ratio is 25 , and the communication range is $30 \mathrm{~m}$. Meanwhile, the estimated error of node coordinate respectively calculated by four algorithms is decreased successively and the estimated error of coordinate simulated on the improved particle swam optimization based on MDV-Hop positioning algorithm is minimized and fluctuation. From the simulation analysis and comparison, the improved particle swam optimization based on MDV-Hop owns the better accuracy in 3D space localization.
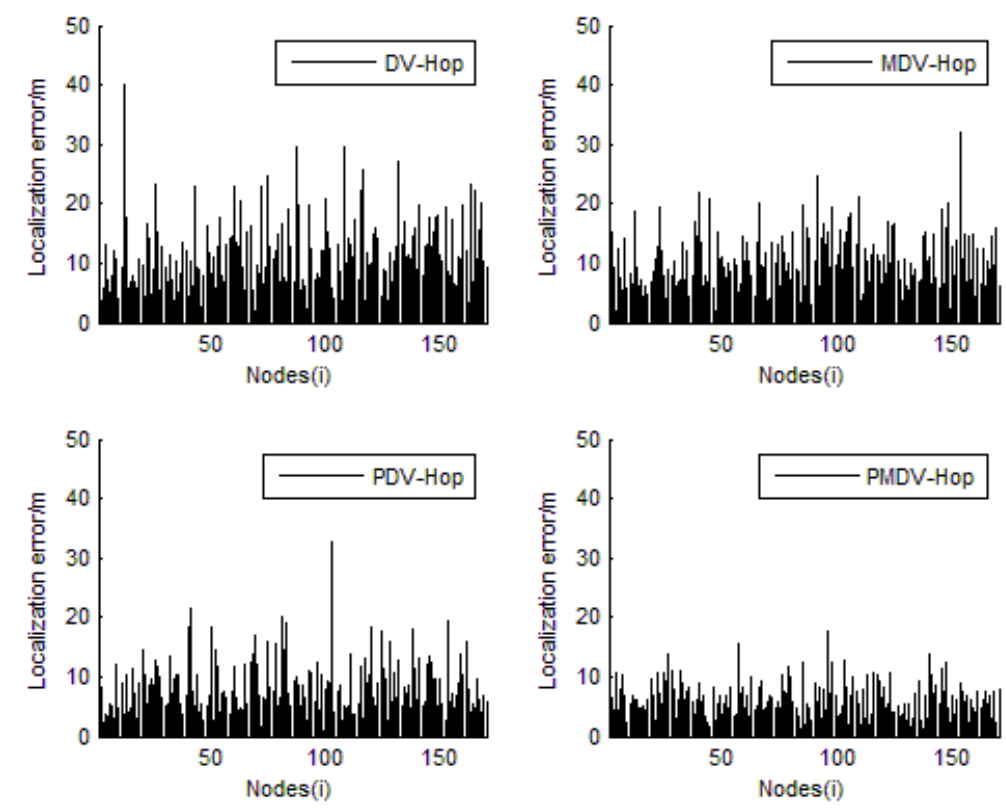

Fig. 8. Comparison of Localization Error based on the DV-hop, MDV-hop, PDV-hop, and PMDV-hop Localization Algorithm

\section{Conclusion}

In this paper, an effective range-free 3D localization scheme was proposed to improve localization accuracy. From the assessment results, it can be inferred that the improved localization algorithm can be applied to obtain more accuracy location information of nodes. The algorithm has been simulated for varied node densities, anchor node ratio and communication range. The analysis of simulations results indicates that (i) as the number of anchor nodes increases, localization error decreases, (ii) as the node density increases, localization error decreases, and (iii) as communication range of nodes in network increases, localization error decreases.

A scheme is applied in the proposed algorithm that the distance per hop dynamically adjusted with weighted value to solve the localization accuracy problem in WSNs. In addition, for optimizing the estimated error of the node coordinate, we present an improved algorithm based on the particle swarm, in which the inertia weight based on concave function is applied 
for the rapid optimization. Meanwhile, due to the performance of trust coefficient, the particles can obtain the optimal solution following their own trajectory and quickly converge to the best location. We have compared PDV-hop, MDV-hop and PMDV-hop with varied parameters of the nodes used in simulation in terms of localization error.

In addition, it will be discussed that the path loss affecting the communication range considering the irregularity radio in localization is very important in our future study. Different simulation scenario would be further analyzed to obtain the influence of the uncertainty of the indicator value on assessment results, for example, the intricate deployment of nodes as mountains. Also, the nodes in the simulations are all considered static without any mobile nodes, so it will be our another step that the mobile nodes is considered in localization work.

\section{Acknowledgments}

This research was supported by the National Natural Science Foundation of China (No.61640020, No.61671244), key research and development program of Jiangsu, China(BE2016368-1), the Agricultural Innovation Program of Jiangsu, China (No. CX(13)3054, CX(14)2114 and CX(16)1006) and the program of Jiangsu Six Talent Peaks (XYDXXJS-033)

\section{References}

[1] S. Samanta, P. U. Tembhare, C. R. Pote, “A Survey on 3d Localization in Wireless Sensor Networks,” IJCSN International Journal of Computer Science and Network, Vol. 2, Issue 1, pp. 48-52, Editorial Board, 2013. Article (CrossRef Link).

[2] S. L. Liu, J. Li, Z. L. Zhang, et al, "Fast and High-Accuracy Localization for Three-Dimensional Single-Particle Tracking,” Scientific reports, 3, 2013. Article (CrossRef Link).

[3] V. K. Chaurasiya, N. Jain, G. C. Nandi, “A novel distance estimation approach for 3D localization in wireless sensor network using multi dimensional scaling,” Information Fusion, 15: 5-18, 2014. Article (CrossRef Link)

[4] S. Lederer, Y. Wang, J. Gao, “Connectivity-based localization of large-scale sensor networks with complex shape,” ACM Transactions on Sensor Networks (TOSN), 5(4): 31, 2009. Article (CrossRef Link)

[5] Q. Shi, H. Huo, T. Fang, et al, "A 3d node localization scheme for wireless sensor networks," IEICE Electronics express, 6(3): 167-172, 2009. Article (CrossRef Link)

[6] D. E. Manolakis, "Efficient solution and performance analysis of 3-D position estimation by trilateration,” Aerospace and Electronic Systems, IEEE Transactions on, 32(4): 1239-1248, 1996. Article (CrossRef Link)

[7] C. Tian, W. Liu, J. Jin, et al, "Localization and synchronization for 3D underwater acoustic sensor networks,” Ubiquitous intelligence and computing, Springer Berlin Heidelberg, 622-631, 2007. Article (CrossRef Link)

[8] R. Mautz, W. Ochieng, G. Brodin, et al, “3D Wireless Network Localization from Inconsistent Distance Observations,” Ad Hoc \& Sensor Wireless Networks, 3(2-3): 141-170, 2007.

Article (CrossRef Link)

[9] Q. Zhang, J. Huang, J. Wang, et al, “A new centralized localization algorithm for wireless sensor network," in Proc. of Communications and Networking in China, ChinaCom 2008, Third International Conference on, IEEE, 625-629, 2008. Article (CrossRef Link)

[10] L. Wang, J. Zhang, D. Cao, “A new 3-dimensional DV-hop localization algorithm,” Journal of Computational Information Systems, 8(6): 2463-2475, 2012. Article (CrossRef Link)

[11] P. K. Singh, B. Tripathi, N. P. Singh, "Node localization in wireless sensor networks," International Journal of Computer Science Engineering \& Information Technology, 2: 2568-2572, 2011. Article (CrossRef Link) 
[12] A. Boukerche, H. A. B. F. Oliveira, E. F. Nakamura, et al, "Localization systems for wireless sensor networks," IEEE Wireless Communications, 14(6): 6-12, 2007. Article (CrossRef Link)

[13] S. Afzal, "A review of localization techniques for wireless sensor networks," Journal of Basic and Applied Scientific Research, 2(8), 7795-7801, 2012. Article (CrossRef Link)

[14] Q. Liu, P. Ren, Z. Zhou, "Three-dimensional Accurate Positioning Algorithm based on Wireless Sensor Networks," Journal of Computers, 6(12): 2582-2589, 2011. Article (CrossRef Link)

[15] W. Cheng, A. Y. Teymorian, L. Ma, et al, "Underwater localization in sparse 3d acoustic sensor networks" in Proc. of INFOCOM 2008, The 27th Conference on Computer Communications, IEEE, 2008. Article (CrossRef Link)

[16] H. Chen, P. Huang, M. Martins, et al, "Novel centroid localization algorithm for three-dimensional wireless sensor networks," in Proc. of Wireless Communications, Networking and Mobile Computing, WiCOM'08, 4th International Conference on, IEEE, 1-4, 2008.

Article (CrossRef Link)

[17] E. Guerrero, W. Hao, J. Alvarez, et al, "A three-dimensional range-free localization algorithm based on mobile beacons for wireless sensor networks," Computer Aided Drafting, Design and Manufacturing, 1: 013, 2010. Article (CrossRef Link)

[18] G. V. Chakaravarthy, S. Marimuthu, A. N. Sait, "Performance evaluation of proposed differential evolution and particle swarm optimization algorithms for scheduling m-machine flow shops with lot streaming," Journal of Intelligent Manufacturing, 24(1), 175-191, 2013.

Article (CrossRef Link)

[19] A. Gopakumar, L. Jacob, "Localization inwireless sensor networks using particle swarm optimization,” in Proc. of IET Int. Conf. Wireless, Mobile Multimedia Netw., pp. 227-230, 2008. Article (CrossRef Link)

[20] R. V. Kulkarni, G. K. Venayagamoorthy, and M. X. Cheng, "Bio-inspired node localization in wireless sensor networks," in Proc. of IEEE Int. Conf. Syst., Man Cybern., San Antonio, TX, pp. 205-210, Oct. 2009. Article (CrossRef Link)

[21] K. S. Low, H. A. Nguyen, H. Guo, "A particle swarm optimization approach for the localization of a wireless sensor network” in Proc. of Industrial Electronics, 2008, ISIE 2008, IEEE International Symposium on, IEEE, 1820-1825, 2008. Article (CrossRef Link)

[22] K. S. Low, H. A. Nguyen, and H. Guo, "Optimization of sensor node locations in a wireless sensor network," in Proc. of 4th Int. Conf. Natural Comput., vol. 5, pp. 286-290, 2008. Article (CrossRef Link)

[23] R. C. Eberhart and Y. H. Shi, "Particle swarm optimization: Developments, applications and resources,” in Proc. of IEEE Congr. Evol. Comput, pp.81-86, Seoul, Korea, 2001. Article (CrossRef Link)

[24] A. Gopakumar, L. Jacob, "Localization in wireless sensor networks using particle swarm optimization,” 2008. Article (CrossRef Link)

[25] R. V. Kulkarni, G. K. Venayagamoorthy, "Particle swarm optimization in wireless-sensor networks: A brief survey [J]," Systems, Man, and Cybernetics, Part C: Applications and Reviews, IEEE Transactions on, 41(2), 262-267, 2011. Article (CrossRef Link)

[26] $\mathrm{H}$. Li, J. Wang, X. Li, “A distributed 3 dimension relative localization algorithm for mobile sensor networks," 2007. Article (CrossRef Link)

[27] C. H. Ou, W. L. He, "Path planning algorithm for mobile anchor-based localization in wireless sensor networks," Sensors Journal, IEEE, 13(2), 466-475, 2013. Article (CrossRef Link)

[28] C. Y. Shih, P. J. Marrón, "COLA: Complexity-reduced trilateration approach for 3D localization in wireless sensor networks," in Proc. of Sensor Technologies and Applications(SENSORCOMM), 2010 Fourth International Conference on, IEEE, 24-32, 2010. Article (CrossRef Link)

[29] W. Qun, P. Ying-Ning, "An improved 3-dimensional mobile location method using volume measurements of tetrahedron,” IEICE transactions on communications, 85(9), 1817-1823, 2002. Article (CrossRef Link)

[30] C. Liu, K. Wu, "Performance evaluation of range-free localization methods for wireless sensor networks" in Proc. of Performance, Computing, and Communications Conference, 2005, IPCCC 2005, 24th IEEE International, IEEE, 59-66, 2005. Article (CrossRef Link) 
[31] F. Belmecheri, C. Prins, F. Yalaoui, L. Amodeo, "Particle swarm optimization algorithm for a vehicle routing problem with heterogeneous fleet, mixed backhauls, and time windows," Journal of Intelligent Manufacturing, 24(4), 775-789, 2013. Article (CrossRef Link)

[32] Z. H. Zhan, J. Zhang, Y. Li, H. H. Chung, "Adaptive particle swarm optimization. IEEE Transactions on Systems, Man, and Cybernetics,” Part B: Cybernetics, 39(6), 1362-1381, 2009. Article (CrossRef Link)

[33] Y. Delice, E. K. Aydoğan, U. Özcan, et al, "A modified particle swarm optimization algorithm to mixed-model two-sided assembly line balancing," Journal of Intelligent Manufacturing, 1-14, 2014. Article (CrossRef Link)

[34] Liu Y, Yang Z, Ning T, et al., "Efficient Quality-of-Service (QoS) Support in Mobile Opportunistic Networks[J],” IEEE Transactions on Vehicular Technology, 63(9), 4574-4584, 2014.

Article (CrossRef Link)

[35] Liu Y, Han Y, Yang Z, et al. "Efficient Data Query in Intermittently-Connected Mobile Ad Hoc Social Networks[J],” IEEE Transactions on Parallel and Distributed Systems, 26(5): 1301-1312, 2015. Article (CrossRef Link)

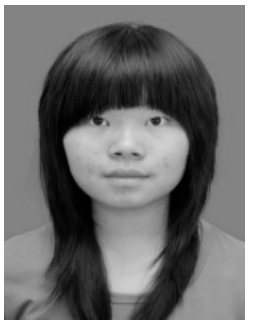

Wenjuan Wang received her BS in network engineering from Nanjing university of science \& technology((NUST)) in 2011. She is currently a student in Nanjing university of science \& technology (NUST)for her MS and PhD .She published one paper in Computer Engineering and Applications. Her research interests include wireless sensor networks and network coding.

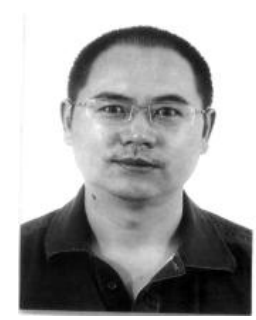

Yuwang Yang received his BS from NorthWestern Polytechnical University in 1988, MS from University of Science and Technology of China in 1991, and Ph.D from Nanj ing University of Science and Technology (NUST)in 1996. From 2000 to2001, he was a visiting scholar in the Department of Computer Science, South Bank University, Londo n. Since 2002, he is an associate professor of Computer Science Department at NUST. H e has published three books on TCP/IP protocol technology, computer network security fields. He has won several provincial and national awards. He is the author of more tha n 30 scientific papers in sensor network and artificial intelligence. He is current on the d uty of several projects on wireless multi-hop network and industry control network. Hi $\mathrm{s}$ research interests are wireless sensor network, industry control network, and intellige nt system. 


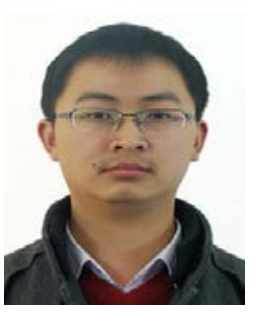

Lei Wang received his bachelor degree from Anhui University of China in 2008. He was a Master-Doctor combined program graduate student in computer science of Nanjing University of Science \&Technology (NUST), China Since 2008. He was a visiting student in Michigan State Uni versity during 2013 - 2014. Currently, he is an assistant professor in School of Computer Science and Technology, Nanjing University of Posts and Telecommunications, China. His research interests are network coding, wireless sensor network, multipath routing, distributed storage system.

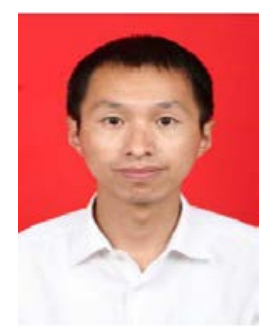

Lu Wei received his bachelor degree from the department of computer science, Shenyang Institute of Aeronautical Engineering, China in 2000, and master degree from Nanjing University of Science \& Technology (NUST) in 2008. Currently, he is studying for his Ph.D degree in NUST. His research interests include wireless communication and information security 